\title{
Sozialgeschichte und histoire culturelle - Perspektiven einer neuen römischen Sozialgeschichte
}

\author{
Thomas Späth E Eckhard Wirbelaner
}

Die methodisch gesicherte Beschreibung antiker Gesellschaften ist kein neues Thema der Geschichtswissenschaft. Doch scheint es mehr denn je geboten, die liebgewonnenen, ja vertrauten Darstellungen zu hinterfragen, zumal diese inzwischen eine ganze Forschergeneration geprägt haben. Dabei waren die klassischen deutschsprachigen Unternehmungen der 1970er Jahre - es sei hier nur an Friedrich Vittinghoffs Handbuch der Europäischen Sozial- und Wirtschaftsgeschichte sowie an Gézy Alföldys Arbeiten erinnert ${ }^{1}$ - keineswegs so unbestritten akzeptiert worden, wie es heute im zeitlichen Abstand scheinen mag. Die Debatte betraf insbesondere die Analyseinstrumente und wurde in den 1980er Jahren bisweilen in recht pointierter Form geführt, nicht zuletzt im SAECULUM. ${ }^{2}$

Neben diesen Auseinandersetzungen traten seit den 1980er Jahren bis dahin unbeachtet gebliebene Fragestellungen und Ansätze immer mehr in den Vordergrund: Die Historische Anthropologie in ganz unterschiedlichen Ausprägungen $^{3}$ oder eine von Grund auf neu konzipierte Religionsgeschichte ${ }^{4}$ ließen die klassischen Diskussionen der sozialgeschichtlichen Forschung in den Hintergrund treten. So kommt es, daß noch heute die meisten Studentinnen und Studenten, die sich eine Vorstellung von Struktur und Entwicklung der römischen Gesellschaft verschaffen wollen, mit Alföldys Synthese arbeiten, obgleich die grundlegend veränderten Sichtweisen der letzten 35 Jahre dort nicht abgebildet sein können.

Der Weg zu einer qualitativ neuen Sicht auf unseren Gegenstand ist freilich

1 Friedrich Vittinghoff (Hg.), Europäische Wirtschafts- und Sozialgeschichte in der römischen Kaiserzeit, Stuttgart 1990 (Handbuch der europäischen Wirtschafts- und Sozialgeschichte 1), bes. $\$ 3$ Gesellschaft: 161-369; ders., Soziale Struktur und politisches System in der hohen römischen Kaiserzeit "O, in: Historische Zeitschrift 230 1980, 31-55 = ders., Civitas Romana. Stadt und politischsoziale Integration im Imperium Romanum der Kaiserzeit, Stuttgart 1994, 253-271; Géza Alföldy, Römische Sozialgeschichte, Wiesbaden 1975, 3. Aufl. 1984, engl. Übersetzung 1988, frz. Übersetzung 1991; ders., Die römische Gesellschaft. Ausgewählte Beiträge, Stuttgart 1986.

2 Karl Christ, Grundfragen der römischen Sozialstruktur, in: Werner Eck, Hartmut Galsterer, Hartmut Wolff (Hg.), Studien zur antiken Sozialgeschichte. Festschrift Friedrich Vittinghoff, Köln/Wien 1980, 197-228; Rolf Rilinger, Moderne und zeitgenössische Vorstellungen von der Gesellschaftsordnung der römischen Kaiserzeit, in: Saeculum 36, 1985, 299-325.

3 Vgl. Jochen Martin, Bedingungen menschlichen Handelns in der Antike. Gesammelte Beiträge zur Historischen Anthropologie, Stuttgart 2009.

${ }_{4}$ Vgl. John Scheid, La religion des Romains, Paris 1998; ders., Quand faire, c'est croire. Les rites sacrificiels des Romains, Paris 2005; J. Rüpke, Von Jupiter zu Christus. Religionsgeschichte in römischer Zeit, Darmstadt im Druck (ersch. 2011/12); Wolfgang Spickermann, Religionsgeschichte des römischen Germanien, 2 Bde. Germania superior/Germania inferior, Tübingen 2003/2008. 
nicht so einfach, gilt es doch nicht nur neue Ansätze, sondern auch verschiedene Wissenschaftskulturen miteinander ins Gespräch zu bringen. Denn auch hier sind in den letzten Jahrzehnten unterschiedliche Diskussionszusammenhänge entstanden, die trotz der oft beschworenen Internationalität des Wissenschaftsbetriebs oft eher unverbunden nebeneinander stehen.

Die im folgenden zum Abdruck gebrachten vier Beiträge wollen in dieser Hinsicht Brücken schlagen und Horizonte eröffnen, und zwar in sprachlicher wie in inhaltlicher Hinsicht. Sie sind im Juni und Oktober 2009 bei Treffen der oberrheinischen Altertumswissenschaftler, die sich im Collegium Beatus Rhenanus zusammengeschlossen haben, vorgestellt und eingehend diskutiert worden. Die nunmehr konstituierte Arbeitsgruppe wird in einem zweiten Schritt versuchen, die methodischen Anregungen auf den Chronotopos Cicero, ${ }^{5}$ insbesondere auf der Basis einer intensiven Auseinandersetzung mit dessen erhaltener Korrespondenz, anzuwenden. Ziel ist es also, an einem absichtlich begrenzten Quellenbestand zu prüfen, inwieweit die angesprochenen sprachlichen wie inhaltlichmethodischen Grenzüberschreitungen unsere Sicht insgesamt neu ausrichten und somit zu einem anderen Verständnis führen können.

Es ist natürlich nicht möglich, in vier Beiträgen die gesamte Dynamik der letzten Jahrzehnte abzubilden. ${ }^{6}$ Doch geben die vier Autoren, jeweils aus ihrem Blickwinkel, wichtige Anregungen für die künftige Arbeit: Michel Humm widmet sich in seinem Beitrag „Histoire culturelle et histoire sociale“ zunächst den verschiedenen Definitionen des Wortes „culture“, um sich dann in einem wissenschaftsgeschichtlichen Zugriff dem unterschiedlichen Gebrauch der heute geradezu inflationär verwendeten Kategorien der Kulturgeschichte, der Cultural Studies und der „histoire culturelle“ anzunähern. Dabei zeigt er nicht nur, wie alle drei Begriffe aus einem spezifischen historischen Kontext heraus entstanden sind, sondern auch jeweils der Abgrenzung von bereits bestehenden Paradigmen

\footnotetext{
5 Was Michail Bachtin zur Unterscheidung unterschiedlicher Roman-Gattungen, die er am Beispiel der antiken Romane entwickelt (Michail M. Bachtin, Formen der Zeit und des Chronotopos im Roman, in: Ders., Formen der Zeit im Roman. Untersuchungen zur historischen Poetik, Frankfurt/ M. 1989, 7-209), wird hier im historischen Zusammenhang umformuliert: Der „Chronotopos Cicero" bezeichnet einen ganz konkret geographischen und zugleich metaphorisch einen sozialen Raum, der in der Zeit und damit in spezifisch historischen Bedingungen situiert ist; Raum und Zeit stehen dabei in einem untrennbaren Verhältnis zueinander und formen das grundlegende Dispositiv der Sozialgeschichte (oder der Geschichte ganz allgemein).

${ }_{6}$ Bei der Tagung in der Römerstiftung Castelen bei Augst war zudem auch Aloys Winterling zugegen, mit dem wir anhand seiner 2001 und 2005 publizierten Beiträge (,Staat', ,Gesellschaft' und politische Integration in der römischen Kaiserzeit, in: Klio 83, 2001, 93-112 sowie ,Öffentlich` und ,privat' im kaiserzeitlichen Rom, in: Tassilo Schmitt, Winfried Schmitz, Aloys Winterling (Hg.), Gegenwärtige Antike - antike Gegenwarten, München 2005, 223-244.) über mögliche Leitbegriffe einer künftigen Synthese zur römischen Sozialgeschichte diskutierten. Gleichfalls anwesend war Fabian Goldbeck, dessen für unser Projekt wichtige Dissertation gerade im Druck erschienen ist: Salutationes. Die Morgenbegrüßungen in Rom in der Republik und der frühen Kaiserzeit, Berlin 2010 (Klio Beihefte NF 16).
} 
dienten. Sein besonderes Augenmerk liegt dabei auf der Entwicklung der französischen Geschichtswissenschaft, die in der „histoire culturelle“ eine mögliche Alternative zum marxistischen Ansatz sah, der zur Erklärung historischer Prozesse zunächst wirtschaftliche Verhältnisse zu verstehen suchte, um dann deren gesellschaftliche Auswirkungen in den Blick zu nehmen, wodurch der "culture“ lediglich der Rang eines Überbauphänomens zugebilligt wurde. Angesichts offensichtlicher Widersprüche, die sich bei diesem Zugriff bei nicht wenigen historischen Gegenständen ergaben - Humm zitiert das Beispiel der französischen Arbeitgeber, die sich 1936/37 der Einführung der 40-Stunden-Woche heftig widersetzten, obgleich diese ihren wirtschaftlichen Interessen durchaus entsprochen hätte.-, entstanden in der französischen Geschichtswissenschaft im Anschluß an die amerikanische Kulturanthropologie (Kroeber - Kluckhohn) Arbeiten, die Sozialgeschichte aus historisch-anthropologischer (und nicht aus wirtschaftsgeschichtlicher) Perspektive erfassen wollten. Zentraler Begriff ist hier die Identität, die unter Rückgriff auf ganz unterschiedliche Elemente Gruppen konstituiert. Dem Historiker ist es dann aufgegeben, die verschiedenen „Repräsentationen“ dieser Identität, ihre Symbole und die hieraus resultierenden Verhaltensweisen und Kommunikationsformen offenzulegen, also eine „histoire sociale des représentations“ (P. Ory) oder eine „histoire des représentations collectives“ (A. Prost) zu schreiben. Eine solchermaßen betriebene „histoire culturelle" ist umfassend in dem Sinne, daß sie sich für jegliche Ausdrucksformen einer Gruppe interessiert, da erst im Zusammenspiel aller dieser Repräsentationen eine genaue Erfassung der Identität möglich ist; sie beansprucht also gegenüber einem klassisch marxistischen oder klassisch sozialwissenschaftlichen Ansatz nicht nur bestimmte, also ausgewählte Phänomene in den Blick zu nehmen, sondern in einem ganzheitlichen Zugriff jenen als defizitär begriffenen Ansätzen überlegen zu sein. Daher gibt es auch hinsichtlich der heranzuziehenden Quellen a priori keine Einschränkung. Allerdings formuliert P. Ory vier allgemeine Regeln, die es bei der Behandlung von Quellen zu beachten gelte, die sich auf die Neutralität des Wissenschaftlers, die räumliche und zeitliche Entstehungssituation der Quelle und ihren Geltungsanspruch beziehen. Im Umgang mit den „kulturellen Hinterlassenschaften“, seien es Artefakte oder menschliche Ideen, wird noch ein wichtiger Unterschied zu früheren Ansätzen deutlich: Nicht das Außergewöhnliche, nicht das Individuelle, nicht das Politische steht im Zentrum dieses „kulturalistischen“ Ansatzes („démarche culturaliste“), sondern das „Typische“, das „Kollektive“ und das „Soziale“. So treten Faktengeschichte, Einzelereignisse und "große Männer" in den Hintergrund, um den Blick auf eine „Menschheitsgeschichte“, eine historische Anthropologie im Sinne von Jacques Le Goff werfen zu können. In diesem Sinne versteht sie sich als Universalgeschichte, die die problematisch gewordene „histoire totale“ abzulösen beansprucht.

Auch Winfried Schmitz redet in seinem Beitrag „Über Grenzen hinweg. Neue sozial- und kulturwissenschaftliche Ansätze in der Alten Geschichte“ einem Per- 
spektivwechsel das Wort. Die klassische sozialgeschichtliche Analyse vermag die großen sozialen Schichten wie Adel, Bauerntum und Unterschicht, die weitgehend aus Sklaven bestand, in den Blick zu nehmen, und doch werden manche aus der Antike überlieferten Berichte erst zum Sprechen gebracht, wenn man Methoden aus weiteren Disziplinen, etwa der Rechtsgeschichte, der vergleichenden Ethnologie der europäischen Kulturen oder der Literaturgeschichte in Anwendung bringt. Exemplarisch läßt sich dies an antiken Beispielen für Rügebräuche (Charivaris) verdeutlichen, die es ermöglichen, die bäuerliche Gesellschaft in ihrer Interaktion zu erfassen. Solche kollektiven Disziplinierungsmaßnahmen sind von willkürlichen und ungerechtfertigen Racheaktionen nicht nur durch die große Zahl der Teilnehmer, sondern auch durch die allgemeine Sichtbarkeit bei der Durchführung der betreffenden Handlung zu unterscheiden. Die Sichtbarkeit dient aber nicht nur dem „Anprangern“ des Gerügten, sondern auch der Wahrung der Grenzen des Rügebrauchs, die dem Gerügten ein Verbleiben in der Gemeinschaft ermöglichte. Da bei den Rügebräuchen die ansonsten geschützten Grenzen des Hauses bewußt und kollektiv verletzt wurden, oblag deren Durchführung zumeist den Ledigen, die selbst noch nicht für eine Hausgemeinschaft verantwortlich waren. So wurde nicht nur vermieden, daß Hausväter die Schutzsphäre fremder Häuser verletzten, sondern es wurden zudem auch noch jungen Mitgliedern dieser Gesellschaft die Normen und das Wertesystem ihrer Gemeinschaft auf drastische Weise vor Augen geführt. „Rügebräuche als kollektiv vollzogene Strafrituale sind charakteristisch für einfach geschichtete, bäuerliche Gesellschaften". Sie werden in einer besonderen kommunikativen Gattung, den mahnenden Sprüchen, Spruchweisheiten und Sprichwörtern tradiert, wie sie uns etwa in den Werken und Tagen des Hesiod begegnen. Wir fassen hier also dank der Sensibilisierung durch andere wissenschaftliche Disziplinen einen Teil der antiken Welt, den bisherige sozial- und wirtschaftsgeschichtliche Methoden nicht in den Blick bekamen. Im Anschluß an dieses konkrete Beispiel bietet W. Schmitz weitere Überlegungen zur „Kulturgeschichte“, die sich als Komplement zu den Ausführungen von M. Humm lesen lassen, da sie vor allem die Diskussionen in der deutschsprachigen Geschichtswissenschaft (O. G. Oexle, R. Vierhaus, S. Tschopp, U. Daniel) vorstellen. Daraus entwickelt er abschließend ein umfangreiches Tableau an Anregungen und Fragen für eine neue römische Sozialgeschichte. Als wegweisend können hier die Arbeiten von Jochen Martin und Egon Flaig genannt werden.

Nach der notwendigen Auseinandersetzung mit dem Historischen Materialismus konnte in den letzten beiden Jahrzehnten bisweilen der Eindruck entstehen, daß der wirtschaftsgeschichtliche Zugriff auf sozialgeschichtliche Fragen völlig diskreditiert sei. Daher haben wir Jean Andreau gebeten, hierzu Stellung zu beziehen. In seinem Beitrag „L'Histoire sociale de Rome dans ses rapports avec l'histoire économique“ bietet er einen wissenschaftsgeschichtlichen Überblick, ausgehend von 5 Bereichen römischer Sozialgeschichte(n): zunächst (1) die So- 
zialgeschichte der beiden führenden ordines, des Senatoren- und des Ritterstandes, dann (2) die weniger angesehenen, gleichwohl recht wohlhabenden Schichten, die wir vor allem dank der Inschriften in den Städten des Römischen Reiches fassen, des weiteren (3) die spezifische Welt des römischen Ägyptens, deren Kenntnis wir vor allem den papyrologischen Quellen verdanken. In allen drei genannten Bereichen kamen prosopographische Ansätze zum Tragen, die durch die Sammlung von Einzelfällen typische Muster erkennen und besser verstehen ließen. Eine nach Andreau „vierte römische Sozialgeschichte“ ließ sich auf der Basis der juristischen Quellen schreiben, während die fünfte das archäologische Fundmaterial in den Blick nahm, genauer, die (beschrifteten und unbeschrifteten) Hinterlassenschaften des täglichen Lebens, wie sie bei Grabungen seit dem 19. Jahrhundert in Massen ans Tageslicht gelangen und von den Archäologen seit den Pionierarbeiten von $\mathrm{H}$. Dressel ausgewertet werden. Natürlich bestehen zwischen diesen verschiedenen römischen Sozialgeschichten mannigfaltige Wechselbeziehungen, doch können diese nicht darüber hinwegtäuschen, daß die fünf Bereiche schon durch ihre unterschiedliche Quellenbasis soweit voneinander getrennt sind, daß es selbst so synthesebegabten Pionieren der antiken Sozial- und Wirtschaftsgeschichte wie etwa Rostovzeff schwer fiel, ein einheitliches Bild zu entwerfen. Vor allem der fünfte Bereich, der jüngste und vielleicht dynamischste, entzieht sich den übrigen vier und bleibt so weitgehend unabhängig; die Kluft scheint sogar in den letzten beiden Jahrzehnten noch größer geworden zu sein. Daß dies aber keineswegs zwingend ist, macht Andreau mit einem wissenschaftsgeschichtlichen Rückblick auf die Grundfrage der wirtschaftsgeschichtlichen Forschung deutlich, die unter dem Namen Bücher-Meyer-Kontroverse bekannt ist: Während Bücher die antike Welt weitgehend von der ,Hauswirtschaft' geprägt sah (was später als ,primitivistische' Position etikettiert wurde), wollten Eduard Meyer (und andere, deren Meinung sich Meyer angeeignet hatte) in Teilen der antiken Wirtschaft moderne Züge erkennen und scheuten sich nicht, Begrifflichkeiten ihrer Zeit (wie „Kapitalismus“ oder „Industrialisierung") bereits auf antike Phänomene anzuwenden. Doch die Debatte blieb zunächst auf den deutschsprachigen Raum beschränkt und wurde erst durch Rostovzeff weiter bekannt gemacht. Seit den Arbeiten von M. I. Finley wurde aus dieser Frage ein Paradigmenstreit, der Wirtschafts- und Sozialgeschichte eng miteinander verband. Für Finley und die Vertreter seiner Sichtweise, die sich als „Primitivisten“ oder „Minimalisten“ von den „Modernisten“, war die römische Wirtschaft kein eigenständiges Phänomen, sondern integrierter Bestandteil der politischen und sozialen Strukturen. Wirtschaftlicher Erfolg war also kein Selbstzweck, sondern untrennbar verbunden mit der sozialen Stellung und ihr unterworfen. Dagegen sahen Wissenschaftler wie J. H. D’Arms in den Unternehmern die eigentliche Führungsschicht des Reiches, die nun mit Macht auf ihre Aufnahme in die ordines drängte. Auch für die Einschätzung der Rolle der Stadt in bezug auf ihr Hinterland ergaben sich konträre Überzeugungen, auch wenn sich 
alle darin einig waren, daß es keinen institutionnellen Unterschied zwischen den Bürger in der Stadt und denen auf dem Lande gab: Für die einen, die Primitivisten in der Nachfolge Finleys, handelte es sich um „die Stadt der Verbraucher“, die auf Kosten des Umlandes lebte, während andere (Ph. Leveau, D. W. Engels, K. Hopkins, auch J. Andreau) der Stadt eine gestalterische Kraft in bezug auf ihr Umland zusprachen. In jedem Fall aber gab es intellektuelle Wechselwirkungen zwischen der Wirtschafts- und der Sozialgeschichte, die für beide Seiten fruchtbar waren. In den letzten Jahren dagegen scheinen diese Wechselwirkungen geringer geworden zu sein, da sich die Wirtschaftsgeschichte mehr den quantitativen Methoden zugewandt hat, etwa im Bereich der Demographie (B. W. Frier, W. Scheidel). Auch die Tendenzen hin zur Neuen Institutionenökonomik („néo-institutionnalisme“), wie sie in der Cambridge Economic History of the Graeco-Roman World erkennbar sind, tragen aus der Sicht von J. Andreau eher zur Abkopplung der Wirtschafts- von der Sozialgeschichte bei. Diesen Strömungen könnte eine Neue römische Sozialgeschichte, die sich für die Einbeziehung wirtschaftsgeschichtlicher Fragestellungen interessiert, entgegenwirken.

Im abschließenden vierten Beitrag führt Thomas Späth in die „Sozialgeschichte mit Geschlecht“ mittels dreier Standortbestimmungen ein: (1) „Geschlecht ist nicht eine deskriptive, sondern eine analytische Kategorie historischer Forschung." Seit der 2. Hälfte der 1980er Jahre wurde der Begriff ,Geschlecht' von der Objektebene („Frauen- und Geschlechterforschung“) auf die methodische Ebene überführt, so daß er nun zur Analyse antiker Quellen über die Beschreibung der spezifischen Lebensbedingungen von Frauen und Männern hinaus benutzt werden kann. Er dient zur Offenlegung von kulturell bestimmten Vorstellungen von Männlichkeit und Weiblichkeit, wie sie uns in antiken Quellen als Bestimmung von Identitäten begegnen. Im Anschluß an die Beiträge von Humm und Schmitz postuliert Späth, daß (2) Geschlecht eine zentrale sozialgeschichtliche Kategorie und (3) ein kulturwissenschaftlicher Begriff sei. Diese Thesen werden in der Erläuterung von Joan Scotts Geschlechtsdefinition im römischen Kontext diskutiert; am Beispiel von Horaz' poetischer Ausgestaltung der Figuren der Kleopatra, des Mark Anton und des Octavian werden die vier Bereiche von Scotts Begriffsbestimmung illustriert: Geschlecht besteht (a) aus kulturell verfügbaren Bildern, die (b) durch normative Konzepte in ihrer Bedeutungsvielfalt eingeschränkt sind und (c) in sozialen Institutionen und Organisationsformen eingeübt werden. Eine auf diese Weise bestimmte Männlichkeit und Weiblichkeit führt zur Erkenntnis der (d) Handlungsräume in der sozialen Konstruktion subjektiver Geschlechtsidentitäten, die es erlaubt, mit Geschlechtsidentitäten zu spielen. Die Kategorie des Geschlechts dient dabei zur Etablierung von Machtbeziehungen innerhalb der betreffenden sozialen Gruppe, es konstruiert und legitimiert Machtverhältnisse und macht diese augenscheinlich. Hieraus ergeben sich verschiedene Bereiche für weitere Forschungsarbeiten, von denen Späth vier ausführt: (1) Vorstellungen von Männlichkeit als Schlüssel zur Erklärung der 
römischen Konzeptualisierung von Macht innerhalb des Hauses (pater familias) sowie die Grenzen dieses Konzepts angesichts der Tatsache, daß nur wenige wirklich die Position des pater familias erreichen können; (2) Wechselwirkungen zwischen Familienidentitäten und subjektiven Identitäten, insbesondere bei Personen, die auch außerhalb der eigenen Familie agieren; (3) Interpretation von politischen Konflikten mit Hilfe der analytischen Kategorie ,Geschlecht' und (4) Geschichte der Regeln sexueller Praktiken als Teil sozialer Umgangsformen, die zur sozialen Konstruktion subjektiver Identitäten beitragen.

Mit diesen geschlechtstheoretischen Thesen, mit den Überlegungen zu den Zusammenhängen zwischen wirtschaftlichem Handeln und gesellschaftlichen Strukturen, den komparatistischen historisch-anthropologischen Ansätzen und den kritischen Anmerkungen zum Nutzen der unterschiedlichen Verwendungen von Kulturbegrifflichkeiten wollen die folgenden vier Beiträge Anregungen vorlegen, wie die in den letzten drei Jahrzehnten entwickelten Perspektiven zu einer neuen Sicht auf die römische Gesellschaft führen können. Sie erheben nicht den Anspruch, Antworten zu geben, sondern werfen Fragen auf. Mit ihrer Publikation knüpft das SAECULUM an eine (zu) lange unterbrochene Debatte an und will zu produktiven Auseinandersetzungen einladen. 